\title{
A Multi-Center International Survey Related to the Nutritional Support after Hematopoietic Stem Cell Transplantation Endorsed by the ASIA Pacific Blood and Marrow Transplantation (APBMT) ${ }^{*}$
}

\author{
Shigeo Fujii", Takehiko Mori², Vincent Lee ${ }^{3}$, Jessica Cheng ${ }^{4}$, Natalie Linton ${ }^{4}$, Albert Lie ${ }^{5}$, \\ Navin Khattry ${ }^{6}$, Akio Shigematsu ${ }^{7}$, Naoyuki Uchida ${ }^{8}$, Tetsuya Eto ${ }^{9}$, Nguyen Duy Thang ${ }^{10}$, \\ Yi-Chang Liu1", Deok-Hwan Yang ${ }^{12}$, Jin Seok Kim ${ }^{13}$, Joon Ho Moon ${ }^{14}$, Dae-Young Kim ${ }^{15}$, \\ Minako Iida $^{16}$, Ritsuro Suzuki ${ }^{16}$, Yoshihisa Kodera ${ }^{17}$, Sung-Won Kim ${ }^{18}$
}

${ }^{1}$ Department of Hematology, University Hospital Wuerzburg, Wuerzburg, Germany; ${ }^{2}$ Division of Hematology, Keio University Hospital, Tokyo, Japan; ${ }^{3}$ Department of Pediatrics, The Chinese University of Hong Kong, Prince of Wales Hospital, Hong Kong (China); ${ }^{4}$ Department of Nutrion Services, St Vincent's Hospital Sydney, Sydney, Australia; ${ }^{5}$ Department of Medicine, Queen Mary Hospital, Hong Kong (China); ${ }^{6}$ Department of Medical Oncology, Tata Memorial Center, Mumbai, India; ${ }^{7}$ Department of Hematology and Oncology, Hokkaido University Hospital, Hokkaido, Japan; ${ }^{8}$ Department of Hematology, Toranomon Hospital, Tokyo, Japan; ${ }^{9}$ Department of Hematology, Hamanomachi Hospital, Fukuoka, Japan; ${ }^{10}$ Hue Regional Hematology and Blood Transfusion Center, Hue Central Hospital, Hue City, Vietnam; ${ }^{11}$ Department of Hematology and Oncology, Kaohsiung Medical University Hospital, Taiwan (China); ${ }^{12}$ Department of Hematology and Oncology, Chonnam National University Hwasun Hospital, Jeollanam-do, Korea; ${ }^{13}$ Division of Hematology, Yonsei University College of Medicine, Severance Hospital, Seoul, Korea (South); ${ }^{14}$ Department of Hematology and Oncology, Kyungpook National University Hospital, Daegu, Korea (South); ${ }^{15}$ Department of Hematology, Asan Medical Center, Seoul, Korea (South); ${ }^{16}$ Department of HSCT Data Management and Biostatistics, School of Medicine, Nagoya University, Nagoya, Japan; ${ }^{17}$ Department of Promotion for Blood and Marrow Transplantation, School of Medicine, Aichi Medical University, Nagakute, Japan; ${ }^{18}$ Department of Hematology and Hematopoietic Stem Cell Transplantation, National Cancer Center Hospital, Tokyo, Japan.



Received December $6^{\text {th }}, 2011$; revised February $1^{\text {st }}, 2012$; accepted February $8^{\text {th }}, 2012$

\begin{abstract}
Background: The nutritional support after hematopoietic stem cell transplantation (HSCT) has not been well established due to the scarcity of clinical trials. To conduct international clinical trials in Asia, we performed the questionnaire survey to investigate the current standard of nutritional support after HSCT. Method: We sent the questionnaire to the physicians nominated by the Asia Pacific Blood and Marrow Transplantation (APBMT) members of each country/ region. Result: We received 15 responses from 7 different countries/regions. The target calorie amount is $1.0-1.3 \times$ basal energy expenditure (BEE) in 11 institutes when partial parenteral nutrition is used. When total parenteral nutrition (TPN) is used, the target calorie amount is $1.0-1.3 \times$ BEE in 9 institutes and $1.3-1.5 \times$ BEE in 4 institutes. Lipid emulsion is routinely used in 12 institutes. Multivitamins and trace elements are routinely added to TPN used in most institutes. It is still uncommon to use the immunonutrition. Blood glucose levels are routinely monitored in all institutes, but the target range varies ( $<110$ in 2 institutes, $<150$ in 4 institutes, and $<200$ in 8 institutes). Conclusions: Basic nutritional support is similar in participating institutes. However, the target glucose level varies and the use of immunonutrition is rather rare. These points can be the theme of future clinical trials.
\end{abstract}

Keywords: Stem Cell Transplantation; Nutritional Support; Asia Pacific Blood and Marrow Transplantation

\section{Introduction}

Hematopoietic stem cell transplantation (HSCT) has now become an integral part in the treatment of hematological diseases, and the use of this treatment has expanded in

${ }^{*}$ There is no conflict of interest to declare.

${ }^{*}$ Corresponding author.
Asia, resulting in the significant increase in numbers of HSCT in Asia as reported [1]. In HSCT, the conditioning regimen including intensive chemotherapy and total body irradiation can potentially cause severe mucositis, nausea, vomiting and diarrhea, which can result in the insufficient oral intake. Even with the use of reduced-intensity 
conditioning regimens, these problems are rather common compared with the use of conventional chemotherapy. Hence, parenteral nutrition is frequently required to maintain the caloric intake. Enteral nutrition (EN) is used with less frequency due to the presence of severe gastrointestinal symptoms such as mucositis and diarrhea in contrast to other treatment modalities in the field of non-surgical oncology except in a few promising reports $[2,3]$. In these situations, intervention by the nutritional support team (NST) is indispensable. However, the management strategy of nutritional support in the field of HSCT has not been well established due to the scarcity of clinical trials compared to other fields. The standards and general recommendations for nutritional support in the field of HSCT are included in the guidelines focusing on the non-surgical oncology by the European Society of Clinical Nutrition and Metabolism (ESPEN) [4]. However, these guidelines were developed mainly following the evidence in other fields of oncology. The beneficial effects of nutritional support after HSCT should be clarified with international large-scale prospective clinical trials. To conduct such international clinical trials in Asia, we established the Working Party Nutritional Support in the Asia Pacific Blood and Marrow Transplantation (APBMT) meeting and performed the questionnaire survey to investigate the current standards prevailing in different Asian institutes.

\section{Materials and Methods}

We sent the questionnaire to the physicians who were nominated by the APBMT members of each country/ region. The questionnaire covered the nutritional practice such as nutritional assessment, use of parenteral nutrition, use of enteral nutrition and glucose control.

\section{Results}

A total of 15 completed questionnaires were received. Fourteen were answered by the physician and one was answered by the dietitian. The characteristics of the hospitals are shown in Table 1.

\subsection{General Information}

A multidisciplinary nutritional support team (NST) in the transplant unit was present in $8(53 \%)$ institutes. The physician, the nurse, the dietitian and the pharmacist were involved in such teams in $75 \%, 75 \%, 100 \%$ and $37.5 \%$, respectively. The activity which NST participates in is pre-transplant nutritional assessment $(75 \%)$, periodic nutritional assessment $(87.5 \%)$, order of parenteral nutrition $(75 \%)$, order of special supplemental,
Table 1. Characteristics of participating institutes.

\begin{tabular}{|c|c|}
\hline Country/region & No. of answers \\
\hline Australia & 1 \\
\hline Hong-Kong & 2 \\
\hline India & 1 \\
\hline Japan & 5 \\
\hline Korea & 4 \\
\hline Taiwan & 1 \\
\hline Vietnam & 1 \\
\hline \multicolumn{2}{|l|}{ Type of hospital } \\
\hline University & 10 \\
\hline Non-university (private) & 1 \\
\hline Non-university (public) & 4 \\
\hline \multicolumn{2}{|c|}{ The number of HSCT case per year } \\
\hline$\geq 200$ cases & 1 \\
\hline $100-199$ cases & 5 \\
\hline 75 - 99 cases & 0 \\
\hline $50-74$ cases & 3 \\
\hline $30-49$ cases & 3 \\
\hline $10-29$ cases & 2 \\
\hline$<10$ cases & 1 \\
\hline
\end{tabular}

nutrition $(87.5 \%)$, order of food in the hospital $(100 \%)$ education of patients about nutrition $(100 \%)$, order of enteral nutriation $(50 \%)$, and order of rehabilitation $(12.5 \%)$.

\subsection{Assessment of Nutritional Status}

Nutritional status is assessed clinically (general appearance, 14 institutes), by caloric intake (12 institutes), anthropometrically ( 2 institutes), biochemically (13 institutes), and by functional capacity (muscle strength, 7 institutes). Serum levels of total protein, albumin, cholinesterase, urine creatinine, pre-albumin, and transferrin are used to assess nutritional status biochemically in 10 , 13, 2, 2, 4 and 1 institutes, respectively.

\subsection{Parenteral Nutrition}

Early total parenteral nutritional (TPN) support is a common practice in all institutes, and TPN is delivered via a central venous (CV) catheter in all institutes. As type of parenteral nutrition, the solution in a 3-chamber bag (glucose, amino acid, and lipid) and the solution in a 
2-chamber bag (glucose, amino acid) are used in 10 and 10 institutes, respectively. TPN is started when patients are unable to take adequate oral intake $(<50 \%-60 \%$ of the target calorie amount) in 6 institutes, is commenced when patients are unable to take adequate oral intake $(<50 \%-60 \%$ of the target calorie amount) and only when it is anticipated that the patient will be unable to eat for more than three days in 2 institutes. TPN is commenced when patients are unable to take adequate oral intake $(<50 \%-60 \%$ of the target calorie amount) and only when it is anticipated that the patient will be unable to eat for more than one week in 4 institutes, is gradually changed from partial parenteral nutrition (PPN) according to the insufficiency of the oral caloric intake in 5 institutes, and is started on the day of transplant in one institute.

Nutritional requirements were set as $1.3-1.5 \times$ BEE (approximately $30-35 \mathrm{kcal} / \mathrm{kg} /$ day) in 4 institutes, 1.0 $1.3 \times \operatorname{BEE}(25-30 \mathrm{kcal} / \mathrm{kg} / \mathrm{day})$ in 9 institutes, and 1.0 $1.2 \times$ measured resting energy expenditure (REE) in 1 institute. One institute uses Schofield Equation. In 12 institutes, fat emulsion is routinely used as a part of TPN including the fat emulsion in all-in-one bag. Fat is given using long-chain triglyceride in 11 institutes, and one institute uses fish-oil based fat emulsion. The percentage of non-protein calories provided by lipid in the institutes using lipid emulsion is $<5 \%$ ( 1 institute), $5 \%-10 \%$ (2 institutes), $10 \%-30 \%$ (7 institutes), and $>30 \%$ ( 2 institutes). The speed of administration is $\leq 0.11 \mathrm{~g} / \mathrm{kg} / \mathrm{h}(8$ institutes), $0.12-0.2 \mathrm{~g} / \mathrm{kg} / \mathrm{h}$ (3 institutes) and $0.21-0.49$ $\mathrm{g} / \mathrm{kg} / \mathrm{h}$ ( 1 institute). Lipid emulsion is not used in 3 institutes due to the perception that it increases the risk of the following factors; infections and liver dysfunction, it is considered unnecessary, and time-consuming. Restrictions imposed by the insurance system in Japan are another reason. Multivitamins and trace elements are routinely added to the TPN solution in 14 institutes (93.3\%) and 13 institutes $(86.7 \%)$, respectively. Glutamine-supplemented parenteral nutrition is used in only 1 institute and fish-oil based parenteral nutrition is used in only 2 institutes.

PPN is used to supplement the oral calorie to maintain the total caloric intake of the patients in 13 institutes (86.7\%). The target calorie amount when PPN was prescribed was set as $1.0-1.3 \times$ calculated BEE (approximately $25-30 \mathrm{kcal} / \mathrm{kg} /$ day) in 11 institutes and 1.0 $1.2 \times$ measured REE in 2 institutes.

\subsection{Enteral Nutrition}

The Enteral nutrition is used in 7 institutes. Enteral nutriation is used in patients with persistent nausea and mucositis after conditioning regimen during neutropenia (3 institutes), persistent nausea after engraftment (in- cluding upper gut graft vs. host disease, 3 institutes), severe side effects with parenteral nutrition (2 institutes), malnutrition with chronic GVHD (6 institutes), and in the intensive care unit (5 institutes). Standard EN is used in 6 institutes, and 1 institute uses semi-elemental EN. Bleeding (1 institute), vomiting (3 institutes) and diarrhea (4 institutes) are serious side effects of EN reported.

\subsection{Glucose Control}

Blood glucose levels are routinely monitored in all institutes. The frequency of the monitoring is daily (10 institutes), three times a week (4 institutes), and two times a week (1 institute). The target glucose level after HSCT is $<110 \mathrm{mg} / \mathrm{dL}$ ( $6.1 \mathrm{mmol} / \mathrm{L}, 2$ institutes), $<150 \mathrm{mg} / \mathrm{dL}$ ( 8.3 $\mathrm{mmol} / \mathrm{l}, 4$ institutes), $<200 \mathrm{mg} / \mathrm{dL}(11 \mathrm{mmol} / \mathrm{L}, 8$ institutes), and in one institute there is no defined target level. Insulin is added to the TPN bag (9 institutes), administered with infusion pump (5 institutes), and used subcutaneously (7 institutes). Eight institutes (53.3\%) are interested in the intensive glucose control (target $80-110$ $\mathrm{mg} / \mathrm{dL}$ ).

\section{Discussion}

This is the first international questionnaire which yielded interesting information about the management of nutriation in the field of HSCT in Asia. The low number of responses to this questionnaire (total 15 institutes) may limit the interpretation of the data. Nevertheless, this information may be of particular interest over the current practice in the absence of firmly established recommenddations for the nutritional support in the field of HSCT.

NST is present in only about a half of institutes. This suggests that patients undergoing HSCT are still not recognized as an established indication for nutritional support in Asia. Another reason could be limitation of human resources in these countries [5].

Nutritional status is mainly assessed clinically, by caloric intake, and biochemically. Nutritional assessment anthropometrically and by functional capacity is not common, even though these assessments are used in other fields [6,7]. The nutritional assessment tools which are useful in other fields should be validated in the HSCT setting.

Parenteral nutrition was the main source of nutritional support after HSCT, which was in contrast to the nutriational support in other fields. In all institutes, TPN was started before patients developed malnutrition [8]. The target dose of TPN was basically within the range of 1.0 $-1.3 \times$ BEE or $1.3-1.5 \times$ BEE which is recommended by various guidelines $[4,9]$. PPN is used to cover the insufficient caloric intake in the majority of the institutes, even though guidelines do not recommend such form of 
nutritional support. Most institutes use the target range of $1.0-1.3 \times \mathrm{BEE}$ when estimating the target caloric intake for patients receiving PPN. Therefore, the target caloric intake with both TPN and PPN was rather homogenous in this questionnaire survey. The target caloric intake of more than $1.0 \times \mathrm{BEE}$ is reasonable to avoid the signifycant weight loss after allogeneic HSCT [10], and less than $1.5 \times \mathrm{BEE}$ is important to avoid the overload of parenteral nutrition which often leads to hyperglycemia and liver damage [11]. Fat emulsion is used as a part of parenteral nutrition in the majority of the institutes, and is administered slowly, especially administered as an all-in-one bag. Soybean lipid emulsion is still the predominate type of fat emulsion used, even though fish-oil based lipid emulsion has promising results in the setting of intensive care units [4]. Multivitamins and trace elements are routinely added to the TPN solution in most institutes. At present, there is only limited experience with supplemented nutrition by new substrates such as glutamine, and omega-3 fatty acids, so-called immunonutrition, in Asia. The use of immunonutrition after transplant is still controversial in Western countries [12, 13]. These nutritional supplements can be the focus of future prospective clinical trials.

Regarding blood glucose monitoring, all institutes monitor glucose level routinely. However, the target range varies significantly from $<110 \mathrm{mg} / \mathrm{dL}$ to $<200 \mathrm{mg} / \mathrm{dL}$. Several retrospective studies showed the association of hyperglycemia and inferior outcomes after allogeneic HSCT [14-17]. Our group previously reported the pilot study of intensive glucose control after allogeneic HSCT, which suggested the possible reduction of infectious diseases with intensive glucose control [18]. In the ICU setting, the benefit of intensive glucose control was not proven by recent large randomized studies [19]. However, after allogeneic HSCT, as mentioned above, the majority of patients receive $\mathrm{PN}$ instead of EN, which is similar to the nutritional support of Leuven study which showed the benefit of intensive glucose control [20]. It is also reported that intensive glucose control in patients with TPN is associated with less hypoglycemia possibly due to the constant administration of glucose solution, which stabilizes the glucose level [21]. On the other hand, EN is associated with frequent interruptions making the administration more unstable which necessi- tates the adjustment of insulin dosage more frequently, this often leads to insufficient glucose control. In addi- tion, several retrospective studies showed that hypergly- cemia in patients receiving TPN is associated with an inferior clinical outcome [22]. Considering the results of our questionnaire survey, there is no consensus regarding the target blood glucose level. Therefore, it will be bene- ficial to perform a clinical trial to assess the beneficial effect of glucose control after allogeneic HSCT.

In conclusion, the answers to this questionnaire demonstrated that the use of parenteral nutrition is the major source of nutritional support after HSCT. Specialized and modified nutritional solutions are still seldom used in Asia. The benefits of these solutions should be assessed by prospective clinical trials. The target range of glucose control varies significantly, which might also be a topic of future clinical trials to determine optimum target blood glucose level after HSCT.

\section{REFERENCES}

[1] A. Yoshimi, R. Suzuki, Y. Atsuta, M. Iida, D. P. Lu, W. Tong, et al., Asia-Pacific Blood and Marrow Transplantation Group (APBMT), "Hematopoietic SCT Activity in Asia: A Report from the Asia-Pacific Blood and Marrow Transplantation Group," Bone Marrow Transplant, Vol. 45, No. 12, 2010, pp. 1682-1691. doi:10.1038/bmt.2010.34

[2] J. Arends, G. Bodoky, F. Bozzetti, et al., DGEM (German Society for Nutritional Medicine), G. Zürcher, R. Fietkau, E. Aulbert, B. Frick, M. Holm, M. Kneba, H. J. Mestrom, A. Zander and ESPEN (European Society for Parenteral and Enteral Nutrition), "ESPEN Guidelines on Enteral Nutrition: Non-Surgical Oncology," Clinical Nutrition, Vol. 25, No. 2, 2006, pp. 245-259. doi:10.1016/j.clnu.2006.01.020

[3] D. Seguy, C. Berthon, J. B. Micol, S. Darré, J. H. Dalle, S. Neuville, et al., "Enteral Feeding and Early Outcomes of Patients Undergoing Allogeneic Stem Cell Transplantation Following Myeloablative Conditioning," Transplantation, Vol. 82, No. 6, 2006, pp. 835-839. doi:10.1097/01.tp.0000229419.73428.ff

[4] F. Bozzetti, J. Arends, K. Lundholm, A. Micklewright, G. Zurcher, M. Muscaritoli and ESPEN, "ESPEN Guidelines on Parenteral Nutrition: Non-Surgical Oncology," Clinical Nutrition, Vol. 28, No. 4, 2009, pp. 445-454. doi:10.1016/j.clnu.2009.04.011

[5] W. W. Souba, "Nutritional Support," New England Journal of Medicine, Vol. 336, No. 1, 1997, pp. 41-8. doi:10.1056/NEJM199701023360107

[6] M. B. Huhmann and D. A. August, "Review of American Society for Parenteral and Enteral Nutrition (ASPEN) Clinical Guidelines for Nutrition Support in Cancer Patients: Nutrition Screening and Assessment," Nutrition in Clinical Practice, Vol. 23, No. 2, 2008, pp. 182-188. doi: $10.1177 / 0884533608314530$

[7] P. B. Soeters, P. L. Reijven, M. A. van Bokhorst-de van der Schueren, J. M. Schols, R. J. Halfens, J. M. Meijers, et al., "A Rational Approach to Nutritional Assessment," Clinical Nutrition, Vol. 27, No. 5, 2008, pp. 706-716. doi:10.1016/j.clnu.2008.07.009

[8] S. A. Weisdorf, J. Lysne, D. Wind, R. J. Haake, H. L. Sharp, A. Goldman, et al., "Positive Effect of Prophylactic Total Parenteral Nutrition on Long-Term Outcome of Bone Marrow Transplantation," Transplantation, Vol. 43, 
No. 6, 1987, pp. 833-838.

[9] M. Martin-Salces, R. de Paz, M. A. Canales, A. Mesejo and F. Hernandez-Navarro, "Nutritional Recommendations in Hematopoietic Stem Cell Transplantation," $\mathrm{Nu}$ trition, Vol. 24, No. 7-8, 2008, pp. 769-775. doi:10.1016/j.nut.2008.02.021

[10] S. Fuji, S. W. Kim, T. Fukuda, S. Kamiya, S. Kuwahara and Y. Takaue, "Positive Impact of Maintaining Minimal Caloric Intake above $1.0 \times$ Basal Energy Expenditure on the Nutritional Status of Patients Undergoing Allogeneic Hematopoietic Stem Cell Transplantation," American Journal of Hematology, Vol. 84, No. 1, 2009, pp. 63-64. doi:10.1002/ajh.21307

[11] P. Sheean and C. Braunschweig, "The Incidence and Impact of Dextrose Dose on Hyperglycemia from Parenteral Nutrition (PN) Exposure in Hematopoietic Stem Cell Transplant (HSCT) Recipients," Journal of Parenteral \& Enteral Nutrition (JPEN), Vol. 30, No. 4, 2006, pp. 345-350. doi: $10.1177 / 0148607106030004345$

[12] M. Crowther, A. Avenell and D. J. Culligan, "Systematic Review and Meta-Analyses of Studies of Glutamine Supplementation in Haematopoietic Stem Cell Transplantation," Bone Marrow Transplant, Vol. 44, No. 7, 2009, pp. 413-425. doi:10.1038/bmt.2009.41

[13] C. Uderzo, P. Rebora, E. Marrocco, S. Varotto, F. Cichello, M. Bonetti, et al., "Glutamine-Enriched Nutrition Does Not Reduce Mucosal Morbidity or Complications after Stem-Cell Transplantation for Childhood Malignancies: A Prospective Randomized Study," Transplantation, Vol. 91, No. 12, 2011, pp. 1321-1325. doi:10.1097/TP.0b013e31821ab959

[14] P. M. Sheean, S. A. Freels, W. S. Helton and C. A. Braunschweig, "Adverse Clinical Consequences of Hyperglycemia from Total Parenteral Nutrition Exposure during Hematopoietic Stem Cell Transplantation," Biol Blood Marrow Transplant, Vol. 12, No. 6, 2006, pp. 656664. doi:10.1016/j.bbmt.2006.01.010

[15] S. Fuji, S. W. Kim, S. Mori, T. Fukuda, S. Kamiya, S. Yamasaki, et al., "Hyperglycemia during the Neutropenic Period Is Associated with a Poor Outcome in Patients
Undergoing Myeloablative Allogeneic Hematopoietic Stem Cell Transplantation," Transplantation, Vol. 84, No. 7, 2007, pp. 814-820. doi:10.1097/01.tp.0000296482.50994.1c

[16] R. L. Derr, V. C. Hsiao and C. D. Saudek, "Antecedent Hyperglycemia Is Associated with an Increased Risk of Neutropenic Infections during Bone Marrow Transplantation," Diabetes Care, Vol. 31, No. 10, 2008, pp. 19721977. doi: $10.2337 / \mathrm{dc} 08-0574$

[17] M. J. Hammer, C. Casper, T. A. Gooley, P. V. O'Donnell, M. Boeckh and I. B. Hirsch, "The Contribution of Malglycemia to Mortality among Allogeneic Hematopoietic Cell Transplant Recipients," Biology of Blood and Marrow Transplantation, Vol. 15, No. 3, 2009, pp. 344-351. doi:10.1016/j.bbmt.2008.12.488

[18] S. Fuji, S. W. Kim, S. Mori, S. Kamiya, K. Yoshimura, H. Yokoyama, et al., "Intensive Glucose Control after Allogeneic Hematopoietic Stem Cell Transplantation: A Retrospective Matched-Cohort Study," Bone Marrow Transplant, Vol. 44, No. 2, 2009, pp. 105-111. doi:10.1038/bmt.2008.431

[19] P. E. Marik and J. C. Preiser, "Toward Understanding Tight Glycemic Control in the ICU: A Systematic Review and Metaanalysis," Chest, Vol. 137, No. 3, 2010, pp. 544-551. doi:10.1378/chest.09-1737

[20] G. van den Berghe, P. Wouters, F. Weekers, C. Verwaest, F. Bruyninckx, M. Schetz, et al., "Intensive Insulin Therapy in Critically Ill Patients," New England Journal of Medicine, Vol. 345, No. 19, 2001, pp. 1359-1367. doi:10.1056/NEJMoa011300

[21] B. R. Bistrian, "Is Total Parenteral Nutrition Protective against Hypoglycemia during Intense Insulin Therapy? A Hypothesis," Critical Care Medicine, Vol. 39, No. 6, 2011, pp. 1533-1535. doi:10.1097/CCM.0b013e31820eb763

[22] P. R. Kumar, P. Crotty and M. Raman, "Hyperglycemia in Hospitalized Patients Receiving Parental Nutrition Is Associated with Increased Morbidity and Mortality: A Review," Gastroenterology Research and Practice, Vol. 2011, 2011, Article ID: 760720, pp. 1-7. 